\title{
LAS CREENCIAS Y EL MUNDO: SOBRE LAS OBJECIONES DE HINTIKKA A QUINE
}

THOMAS M. SIMPSON

Consejo Nacional de Investigaciones Científicas de la Argentina

\section{Introducción}

En "Quantifiers and Propositional Attitudes" (1956), Quine distingue entre los sentidos 'nocional' y 'relacional' de los operadores psicológicos; esta distinción reaparece en $W$ ord and Object (1960), bajo otro ropaje verbal, como la oposición entre el sentido 'opaco' y el sentido 'transparente' de los verbos de actitudes proposicionales. La interpretación opaca fue tema central de la semántica de orientación lógica desde Frege, debido al hecho escandaloso de que los contextos opacos resisten la aplicación de reglas lógicas elementales, como la sustituibilidad de los idénticos y la generalización existencial. Salvo alguna excepción notable, la presencia tenaz de oraciones psicológicas cuyo análisis exige la aplicabilidad de esas reglas fue pasada por alto. De Frege a Carnap, las oraciones psicológicas fueron condenadas a la opacidad total; en el capítulo de Meaning and Necessity (1947) dedicado a los contextos de creencia, Carnap ni siquiera menciona la posibilidad de un análisis distinto. Esta situación cambia con la publicación de "Quantifiers and Propositional Attitudes", que recupera del olvido la interpretación transparente, llama la atención sobre su extraordinaria importancia y propone un artificio notacional destinado a facilitar su manipulación lógica.

En las notas que siguen examino dos objeciones de Jaakko Hintikka a la propuesta de Quine; me propongo mostrar que se originan en una apreciación incorrecta de las posibilidades que ofrece la notación quineana, cuya flexibilidad y 
fuerza expresiva son mucho mayores de lo que sugiere una lectura polémica.

Por razones que omito aquí, usaré la dicotomía 'de dicto de $r e$ ' en lugar de la distinción en términos de 'opacidad' versus 'transparencia', si bien los textos citados conservan su terminología original.

\section{El caso de Pepe el Pistolero}

Consideremos las tres oraciones siguientes:

(A) $\sim(\exists x)$ (la policía cree que el banco fue asaltado por $x)$;

(B) La policía cree que el banco fue asaltado por el hombre al que vieron manejando un sedán gris;

(C) Pepe el Pistolero = el hombre al que vieron manejando un sedán gris.

Según Hintikka, (A)-(C) pueden ser conjuntamente verdaderas. ${ }^{1}$ Hintikka supone que, en concordancia con (A), la policía no sabe quién es el hombre que fue visto manejando un sedán gris "ni tiene opinión alguna sobre el asunto", y que Pepe el Pistolero es un viejo conocido de la policía, aunque ésta no abriga ninguna sospecha acerca de él (loc. cit., p. 144).

Estos son los datos del problema, tal como los presenta Hintikka; sobre la base de ellos, su objeción es formulable así: "Quine estaría obligado a decir que, en el sentido en el cual la oración [Hintikka se refiere aquí a (B); T.M.S.] puede ser generalizada existencialmente" (loc. cit.), es verdad, pese al desconocimiento indicado, que

1 Knowledge and Belief (en adelante: "KB"), Ithaca, New York, Cornell University Press, 1962, p. 144. Aunque Hintikka introduce su ejemplo de manera informal, supongo que mi sistematización no distorsiona sus intenciones. Una versión castellana del terto de Hintikka que examinamos en este trabajo puede hallarse en: Thomas M. Simpson, Semántica filosófica: problemas y discusiones, Buenos Aires-Madrid, Siglo XXI Argentina Editores, 1973, pp. 231-233. 
(D) La policía cree que el banco fue asaltado por Pepe el Pistolero; y

(E) ( $\exists x$ ) (la policía cree que el banco fue asaltado por $x$ ),

donde (D) se obtiene a partir de (B) por sustitución (en virtud de (C)), y (E) a partir de (D) por generalización existencial.

Pero es obvio que (E) contradice (A), y lo mismo ocurre con (D), que implica (E). En consecuencia, la teoría de Quine debe contener algo erróneo. Por otra parte, existe un claro sentido intuitivo de acuerdo con el cual (A), (B) y (C) pueden ser conjuntamente verdaderas; la situación de Quine parece, pues, del todo insostenible.

Notemos, sin embargo, que las oraciones psicológicas pueden interpretarse tanto de re como de dicto, y que, en consecuencia, la posibilidad de la verdad conjunta de (A), (B) y (C) depende de cuál sea la interpretación elegida. Desde el punto de vista de Quine, (A) sólo puede ser interpretada de re; pero ¿qué ocurre con (B)? La validez de la objeción de Hintikka requiere que la interpretación sea también de $r e$ en este caso, pues es precisamente tal interpretación la que se pone en tela de juicio.

Pero si (B) recibe una interpretación de re entonces es cuestionable la consistencia misma de (A) $-(\mathrm{C})$, y precisamente porque (B) y (C) implican (E), que contradice (A). Podríamos decir que si (A) y (C) son verdaderas, entonces (B) es falsa en la interpretación de re, la cual permite inferir (D) y $(E) ;^{2}$ sea como fuere, Quine no está obligado a aceptar que, con tal interpretación, (B) sea verdadera. Sostendremos aquí que (B) parece compatible con (A) porque se la entiende de dicto, y que es debido a ello que la inferencia que conduce a (D) resulta intuitivamente persuasiva.

2 No discutimos aquí la llamada "regla de exportación", que nos permitiría inferir (D) -interpretada de re- a partir de una interpretación de dicto de (B), y la premisa adicional de que Pepe el Pistolero existe. La regla de exportación fue introducida por Quine en "Quantifiers and Propositional Attitudes" y abandonada posteriormente. Para una versión castellana del artículo mencionado véase también T. M. Simpson, Semántica filosófica. 
Comencemos con este último paso inferencial. En la notación quineana para la abstracción intensional, la deducción de (D) puede representarse como sigue (dejando (C) tal como está):

$\left(B^{\prime}\right)$ La policía cree ( $x$ [la persona que asaltó el banco $=x]$, el hombre al que vieron manejando un sedán gris);

(C) Pepe el Pistolero = el hombre al que vieron manejando un sedán gris;

$\left(D^{\prime}\right)$ La policía cree ( $x$ [la persona que asaltó el banco $=x]$, Pepe el Pistolero).

Admito que podemos sentir que hay todavía algo extraño en el tránsito de $\left(B^{\prime}\right)$ y $(C)$ a $\left(D^{\prime}\right)$; al ser consultada sobre el asunto, la policía podría rechazar $\left(\mathrm{D}^{\prime}\right)$, pero aceptar $\left(\mathrm{B}^{\prime}\right)$.

Quizás sea útil recordar aquí la distinción entre lo que Nicholas Rescher ha llamado "el lenguaje de la fuente" (el de la persona que afirma la oración de creencia) y "el lenguaje del sujeto" (el de la persona a quien se atribuye la creencia). ${ }^{8}$ Dentro de la teoría de Quine se supone que la atribución a un sujeto de una creencia de re concerniente a un objeto dado sigue siendo verdadera sea cual fuere el nombre usado por la fuente para referirse a ese objeto. En $\left(B^{\prime}\right)$ y $\left(\mathrm{D}^{\prime}\right)$ somos nosotros (la fuente) y no la policía (el sujeto) quienes usamos las palabras "El hombre al que vieron manejando un sedán gris" y "Pepe el Pistolero" para hacer refe. rencia a la misma persona. $\mathrm{Si}\left(\mathrm{B}^{\prime}\right)$ es verdadera, también lo es $\left(\mathrm{D}^{\prime}\right)$; y si la policía rechaza $\left(\mathrm{D}^{\prime}\right)$, sólo mostrará con ello que si bien cree $x$ [la persona que asaltó el banco $=x$ ] de Pepe el Pistolero, no cree sin embargo $x$ [x es llamado "Pepe el Pistolero"] de Pepe el Pistolero."

Volvamos ahora a la supuesta consistencia de (A)-(C).

3 N. Rescher, "The Problem of a Logical Theory of Belief Sentences" (Cap. V de Topics in Philosophical Logic, Dordrecht-Holland, D. Reidel Publishing Co., 1968; versión castellana incluida en: T. M. Simpson, Semántica filosófica). Pero recuérdese que no es necesario que el sujeto hable nuestro lenguaje, o un lenguaje cualquiera.

4 Volveremos a este punto en la sección 3. 
Aunque $\left(B^{\prime}\right)$ no es la única posible interpretación de re que puede darse a (B), es sin duda la más plausible. ${ }^{5}$ Pero, como se ha sugerido antes, puede sostenerse que es falsa porque implica la oración falsa

$\left(\mathrm{E}^{\prime}\right)$ ( $\left.\exists y\right)$ (La policía cree $x$ [la persona que asaltó el banco $=x], y)$.

Por lo tanto, la consistencia de (A)-(C) requiere una interpretación de dicto de (B), la cual podría ser simplemente

(B*) La policía cree [la persona que asaltó el banco $=\mathrm{el}$ hombre al que vieron manejando el sedán gris].

Para inferir $\left(E^{\prime}\right)$ necesitaríamos la regla de exportación, cuyo examen es independiente del tema que discutimos.

\section{El caso del dictador de Portugal}

El principal argumento de Hintikka relativo a este ejemplo depende de su análisis de la noción de 'saber quién', de acuerdo con el cual "Juan sabe quién mató a Rosalía" sig. nifica lo mismo que " $(\exists x)$ (Juan sabe que $x$ mató a Rosalía)" ( $K B$, pp. 131-132). Según Hintikka, puede mostrarse que la teoría de Quine es insatisfactoria "siempre que acep. temos el análisis de la frase 'saber quién' esbozado en la sección 6.3" (loc. cit., p. 143).

Consideremos la siguiente sucesión de oraciones:

(1) Juan sabe que el dictador de Portugal = el dictador de Portugal;

(2) 'El Dr. Salazar = el dictador de Portugal;

(3) Juan sabe que el dictador de Portugal = el doctor Salazar;

(4) $(\exists x)$ (Juan sabe que el dictador de Portugal $=x$ ).

5 Las restantes son: $a$ ) La policía cree ( $x$ [el hombre al que vieron manejando un sedán gris $=x]$, la persona que asaltó el banco); b) La policía cree $(x, y[x=y]$, la persona que asaltó el banco, el hombre al que vieron manejando un sedán gris). 
Hintikka afirma que en la teoría de Quine (1) "admite dos interpretaciones, una transparente y una opaca" (p. 142) y que para la verdad de (1) "basta que Juan sepa que Portugal es una dictadura" (p. 143). ${ }^{6}$ Examina entonces la inferencia de (1) y (2) a (3):

Puesto que (3), cuando se la interpreta de modo transparente es implicada por (1), debe ser también verdadera si lo es (1), incluso si nuestro hombre no tiene la menor idea acerca del nombre u otros atributos del dictador de Portugal. Esto puede parecer desconcertante, pero probablemente pueda eliminarse como un curioso subproducto de la dualidad de interpretaciones: lo que es verdadero en una interpretación no tiene por qué serlo en la otra (loc. cit., p. 143; la bastardilla es mía).

En este punto se puede detectar fácilmente el origen del error: se halla en los supuestos iniciales de que hay sólo dos interpretaciones quineanas de (1) y que (1) es equivalente a "Juan sabe que Portugal es una dictadura". ${ }^{\text {. En realidad }}$ (1) admite una interpretación de dicto y dos interpretaciones de re:

(i) Juan sabe [el dictador de Portugal $=$ el dictador de Portugal];

(ii) Juan sabe $(x[$ el dictador de Portugal $=x]$, el dictador de Portugal);

(iii) Juan sabe $(x, y,[x=y]$, el dictador de Portugal, el dictador de Portugal).

Basta una mirada a (ii) para rechazar el supuesto de que (1) equivale a la afirmación de que Juan sabe que Portugal es una dictadura, pues Juan podría estar enterado de este

- He cambiado la numeración y los nombres; la cita no es, pues, tertual en relación con estos detalles.

7. En su artículo "Partially Transparent Senses of Knowing" (Philosophical Studies, Vol. XX (1969), números 1/2, p. 8), Hintikka abandona el segundo supuesto. 
hecho aunque (ii) fuera falso. Como ya lo hemos señalado en relación con el caso de Pepe el Pistolero, no hay nada desconcertante en la inferencia de (3) a partir de (1)-(2) si (1) es interpretada como (ii) y (3) como

(3') Juan sabe $(x[$ El dictador de Portugal $=x]$, el Dr. Salazar).

Es importante insistir en que no es cierto que esta inferencia pueda realizarse "incluso si nuestro hombre no tiene la menor idea acerca del nombre $\mathbf{u}$ otros atributos del dictador de Portugal": de acuerdo con (ii) Juan sabe, acerca del dictador de Portugal (sea cual fuere el nombre que use para designarlo, si es que usa alguno), que él posee el atributo de ser el dictador de Portugal: vale la pena recordar que $x$ [el dictador de Portugal $=x]$ es el objeto de la 'actitud' epistémica de Juan y que tal objeto es una intensión. ${ }^{8}$

Pero es ya tiempo de examinar la objeción principal de Hintikka:

Lo que es inaceptable es que (4) sea implicada por (3) y por lo tanto [a través de (2)] por (1), interpretada de manera transparente. Puesto que la última oración es trivialmente verdadera, la primera [o sea (4)] debe serlo también. Se sigue que nadie puede dejar de saber quién es el dictador de Portugal si sabe que Portugal es una dictadura. La dualidad de interpretaciones ya no nos ayuda en este caso, pues [...] según Quine la generalización existencial con respecto a un término sólo es posible cuando el término figura en un contexto transparente. En consecuencia, el operador epistémico de (4) debe ser interpretado de manera transparente. Pero entonces (4) es im. plicada por (1), al igual que por (2). [...] Puesto que no puedo encontrar ningún defecto en mi análisis de la

8 Este es sólo uno de los procedimientos admitidos por la notación canónica de Quine, quien repudia las intensiones; empleamos la abstracción intensional porque hace intuitivamente más claro el punto discutido, que es independiente de las opciones ontológicas. 
frase 'sabe quién' [...] concluyo que debe haber algo erróneo en la concepción de Quine (p. 143; la bastardilla es mía). ${ }^{\ominus}$

Por impresionante que pueda parecer, este argumento se basa en un malentendido acerca de la posición de Quine. En primer lugar, digamos algunas palabras acerca de la "trivialidad" de (1). Es claro que (1) parece trivial sólo si suponemos que todas las figuraciones de términos singulares en (1) son puramente referenciales. Pero ya vimos que el análisis quineano de las actitudes de re obliga a considerar (1) como ambiguo, puesto que puede recibir las interpretaciones (ii) y (iii). Y si bien podría decirse que (iii) transmite una información 'trivial', no es posible afirmar lo mismo sobre (ii); esta última oración implica

(4') $(\exists y)$ (Juan sabe $(x[$ el dictador de Portugal $=x], y)$, que ciertamente transmite esa "información apremiante" que Quine atribuyó a (4), ${ }^{10}$ en contraste evidente con la generalización existencial de (iii):

(5) $(\exists w)(\exists z)$ (Juan sabe $x, y[x=y], w, z)$,

la cual se reduce a la afirmación de que existe alguien acerca de quien Juan sabe que es idéntico a sí mismo.

Enfoquemos ahora nuestra atención en la parte del argumento que depende del análisis de 'saber quién' formulado por Hintikka. Considerando el carácter problemático de esta noción, la objeción de Hintikka podría responderse mediante

$\theta$ Hintikka piensa que "el sentido opaco de conocer es el sentido normal de esta noçión, inclusive cuando figura en un contexto cuantificacional" ("Individuals, Possible Worlds and Epistemic Logic", NOOS, Vol. 1, No. 1 (1967), p. 34, nota). Hemos caído, al parecer, en una trampa ineludible, porque el quid de la interpretación transparente dada por Quine a (4) es hacer posible la expresión de esa "información apremiante" que según él no puede ser expresada cuando el contexto gobernado por "saber" es opaco.

10 "Véase la información apremiante que transmite la oración 'Hay alguien de quien yo creo que es un espía' en contraste con 'Yo creo que hay alguien que es un espía' (en el sentido débil de 'Yo creo que hay espías')" (Word and Object, p. 148). 
el simplé rechazo de su análisis $;{ }^{11}$ pero también es posible responder que, cuando (1) recibe una interpretación de re, el hecho de implicar que Juan sabe quién es el dictador de Portugal deja de ser tan desconcertante como Hintikka pretende. Por supuesto, sería realmente escandaloso que la teoría de Quine implicara que "nadie puede dejar de saber quién es el dictador de Portugal si sabe que Portugal es una dictadura"; recordemos, sin embargo, que (1) puede recibir dos interpretaciones de re (las anteriores (ii) y (iii)) y que ninguna de ellas equivale a la afirmación de que Juan sabe que hay una dictadura en Portugal. Es posible admitir que algún tipo de 'saber quién' constituye una condición necesaria para la existencia de una actitud de re; en tal caso tendremos, por ejemplo, que

(iv) Si Juan sabe ( $x$ [el dictador de Portugal $=x]$, el dictador de Portugal), entonces Juan sabe quién es el dictador de Portugal,

por elusivos que puedan ser los criterios de aplicabilidad de la noción 'saber quién'. ${ }^{12}$ Pero ésta es una cuestión abierta, perfectamente compatible con el enfoque de Quine, y digna de ser explorada por sí misma. Después de todo, Quine nun. ca dijo poseer criterios generales para afirmar la existencia de actitudes de re en casos específicos; se limitó a proponer un análisis de algunos aspectos de su lógica. Si tenemos presente que la regla de exportación es un agregado prescindible en la teoría de Quine, ${ }^{13}$ podríamos avanzar un paso más y sostener inclusive que Quine dio en el clavo al poner de relieve un rasgo importante de las actitudes de re que las distinguen de las de dicto: el hecho de que las primeras implican alguna forma de 'saber quién'.

11 Ver Héctor-Neri Castañeda, "Thinking and the Structure of the World" (Philosophia, Vol. 4, No. 1 (1974), pp. 33-35).

12 "Los criterios acerca de cuándo puede decirse que sabemos quién es éste o aquél hombre son altamente variables. $A$ veces es suficiente conocer el nombre de la persona en cuestión" (J. Hintikka, $K B$, p. 149, nota 9).

13 Cf. la respuesta de Quine a Sellars en Synthese, Vol. 19, No. 1/2 (1968), pp. 307-308. 
In "Quantifiers and Propositional Attitudes" (1956), Quine makes a distinction between the 'notional' and 'relational' meanings of the psychological operators. This distinction reappears, in different garb, in Word and Object (1960), this time as between the opaque and transparent senses of expressions of propositional attitudes. The opaque interpretation has been the central theme in semantics with a logical slant since Frege, due to the fact that the opaque contexts resist the application of elementary logical rules, such as the sub. stitutivity of identity and existential generalization. Save some notable exceptions, the tenacious presence of psychological sentences whose analysis requires the applicability of these rules was overlooked. From Frege to Carnap, psychological expressions were condemned to total opacity; in the chapter of Meaning and Necessity (1947) devoted to 'sentences about beliefs', Carnap did not even mention the possibility of a different analysis. This situation changes with the publication of "Quantifiers and Propositional Attitudes," which rescues the transparent interpretation from oblivion, calls attention to its extraordinary importance, and proposes a notational device bound to facilitate its logical manipulation.

In this article, I examine two objections made by Jaako Hintikka to Quine's proposal. I intend to show that they are based on an incorrect evaluation of the possibilities offered by Quinean notation, whose flexibility and expressive strength are far greater than what a polemic reading might suggest. For reasons $I$ will omit here, I use the 'de dicto - de re' dichotomy instead of the distinction in terms of 'opacity' and 'transparency', although the texts quoted keep their original terminology.

(Summary by Thomas M. Simpson) 